\title{
Three-port beam splitters-combiners for interferometer applications
}

\author{
R. Schnabel, A. Bunkowski, O. Burmeister, and K. Danzmann \\ Max-Planck-Institut für Gravitationsphysik (Albert-Einstein-Institut), Universität Hannover, \\ Callinstrasse 38, 30167 Hannover, Germany
}

\begin{abstract}
Received September 19, 2005; accepted November 3, 2005; posted November 29, 2005 (Doc. ID 64890)
We derive generic phase and amplitude coupling relations for beam splitters-combiners that couple a single port with three output ports or input ports, respectively. We apply the coupling relations to a reflection grating that serves as a coupler to a single-ended Fabry-Perot ring cavity. In the impedance-matched case such an interferometer can act as an all-reflective ring mode cleaner. It is further shown that in the highly undercoupled case almost complete separation of carrier power and phase signal from a cavity strain can be achieved. (c) 2006 Optical Society of America

OCIS codes: $050.1950,120.3180,230.1360$.
\end{abstract}

Two-port beam splitters-combiners, for example, partially transmitting mirrors, are key devices in laser interferometry. They serve as 50/50 beam splitters in Michelson interferometers and as low transmission couplers to cavities. Amplitude and phase relations of two-port beam splitters-combiners are well known. In the case of grating optics, diffraction orders of a greater number can couple to one input port. Recently a reflection grating with three diffraction orders was used for interferometer purposes; laser light was coupled into a linear high-finesse Fabry-Perot cavity by using the second-order Littrow configuration. ${ }^{1}$ The grating was built from a binary structure. This property, together with the secondorder Littrow configuration, provided a symmetry against the grating's normal. The system was theoretically analyzed in Ref. 2 . It was shown that a new three-port (3p) coupled Fabry-Perot interferometer can be designed such that resonating carrier light is completely backreflected toward the laser source. The additional interferometer port is then on a dark fringe and contains half of the interferometer strain signal.

In this Letter we first derive the generic coupling relations of $3 p$ beam splitters. This includes coupling amplitudes and coupling phases that are required for interferometric applications. Our description includes arbitrary gratings with three orders of diffraction regardless of the groove shape and the diffraction angles, as shown in Fig. 1. We then investigate the $3 p$ reflection grating coupled Fabry-Perot ring interferometer and show that for a resonating carrier a dark port can be constructed that contains an arbitrary high fraction of the interferometer's strain signal.

Optical devices can be described by a scattering matrix formalism. ${ }^{3}$ In general the coupling of $n$ input and $n$ output ports requires an $n \times n$ scattering matrix $\mathbf{S}$. The $n$ complex amplitudes of incoming and outgoing fields are combined into vectors $\mathbf{a}$ and $\mathbf{b}$, respectively. For a lossless device $\mathbf{S}$ has to be unitary to preserve energy, and reciprocity demands $\left|S_{i j}\right| \equiv\left|S_{j i}\right|$ for all elements $S_{i j}$ of $\mathbf{S}$. For a generic 3p device six coupling amplitudes and nine coupling phases are involved. Since three input and three output fields are considered, the number of phases can be reduced to six without loss of physical generality; the remaining six phases describe the phases of the six fields with respect to a local oscillator field. Here we choose the phases such that the matrix $\mathbf{S}$ is symmetric, and $\mathbf{b}$ $=\mathbf{S} \times \mathbf{a}$ can therefore be written as

$$
\left(\begin{array}{l}
b_{1} \\
b_{2} \\
b_{3}
\end{array}\right)=\left(\begin{array}{ccc}
\eta_{1} e^{i \phi_{1}} & \eta_{4} e^{i \phi_{4}} & \eta_{5} e^{i \phi_{5}} \\
\eta_{4} e^{i \phi_{4}} & \eta_{2} e^{i \phi_{2}} & \eta_{6} e^{i \phi_{6}} \\
\eta_{5} e^{i \phi_{5}} & \eta_{6} e^{i \phi_{6}} & \eta_{3} e^{i \phi_{3}}
\end{array}\right) \times\left(\begin{array}{c}
a_{1} \\
a_{2} \\
a_{3}
\end{array}\right),
$$

where $0<\eta_{i}<1$ for all $i$ describes the amplitude and $e^{i \phi_{i}}$ describes the phase of coupling. Figure 1 shows
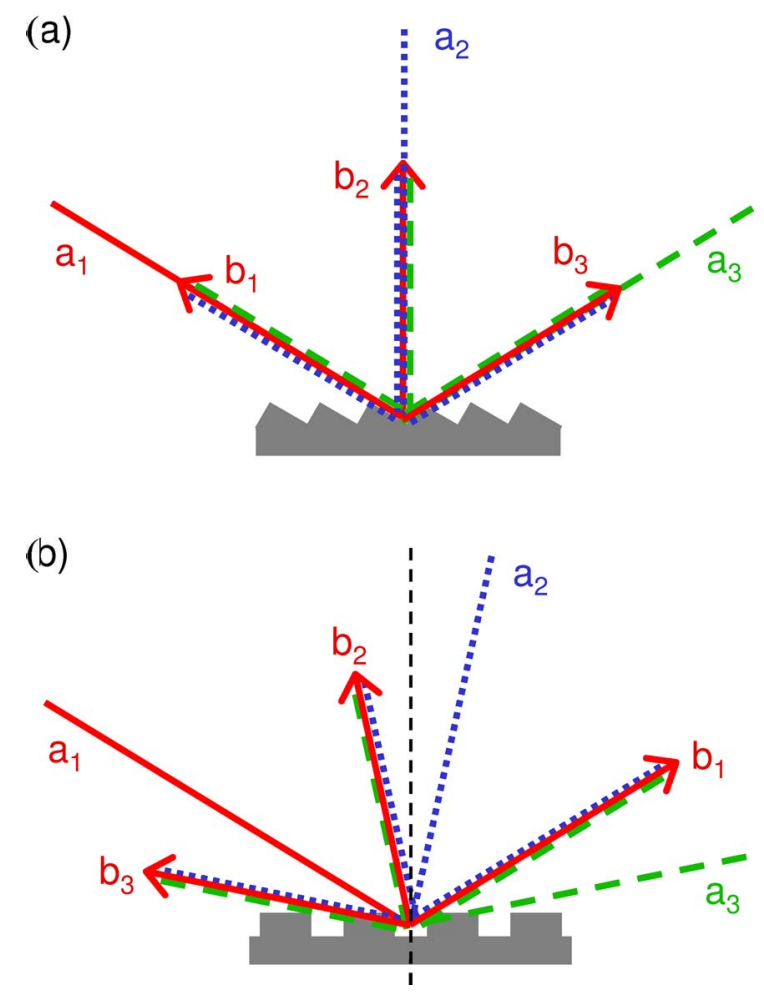

Fig. 1. (Color online) Two examples of the three-port (3p) beam splitters and/or combiners. Input fields $a_{i}$ and output fields $b_{i}$ denote complex amplitudes of the electric field. (a) Asymmetric triangular grating in second-order Littrow configuration. (b) Binary grating in non-Littrow configuration. 
two examples of $3 p$ devices. In both cases the input beam splits into three beams, and, vice versa, three input beams can interfere to become a single one. However, one realizes that the rigorously defined scattering matrix for the device in Fig. 1(b) has a dimension of $6 \times 6$, but this matrix contains null elements because not six but only three ports couple, and the matrix can be reduced to the matrix as given in Eq. (1).

The unitarity condition $\mathbf{S}^{\dagger} \mathbf{S}=\mathbf{1}$ entails the following set of equations:

$$
\begin{aligned}
1 & =\eta_{1}^{2}+\eta_{4}^{2}+\eta_{5}^{2} \\
1 & =\eta_{2}^{2}+\eta_{4}^{2}+\eta_{6}^{2} \\
1 & =\eta_{3}^{2}+\eta_{5}^{2}+\eta_{6}^{2} \\
\left|\cos \left(2 \phi_{4}-\phi_{1}-\phi_{2}\right)\right| & =\frac{\left|\eta_{5}^{2} \eta_{6}^{2}-\eta_{1}^{2} \eta_{4}^{2}-\eta_{2}^{2} \eta_{4}^{2}\right|}{2 \eta_{4}^{2} \eta_{1} \eta_{2}}, \\
\left|\cos \left(2 \phi_{5}-\phi_{1}-\phi_{3}\right)\right| & =\frac{\left|\eta_{4}^{2} \eta_{6}^{2}-\eta_{1}^{2} \eta_{5}^{2}-\eta_{3}^{2} \eta_{5}^{2}\right|}{2 \eta_{5}^{2} \eta_{1} \eta_{3}} \\
\left|\cos \left(2 \phi_{6}-\phi_{2}-\phi_{3}\right)\right| & =\frac{\left|\eta_{4}^{2} \eta_{5}^{2}-\eta_{2}^{2} \eta_{6}^{2}-\eta_{3}^{2} \eta_{6}^{2}\right|}{2 \eta_{6}^{2} \eta_{2} \eta_{3}}, \\
\left|\cos \left(\phi_{6}+\phi_{4}-\phi_{5}-\phi_{2}\right)\right| & =\frac{\left|\eta_{1}^{2} \eta_{4}^{2}-\eta_{2}^{2} \eta_{4}^{2}-\eta_{5}^{2} \eta_{6}^{2}\right|}{2 \eta_{2} \eta_{4} \eta_{5} \eta_{6}}, \\
\left|\cos \left(\phi_{6}-\phi_{4}-\phi_{5}+\phi_{1}\right)\right| & =\frac{\left|\eta_{3}^{2} \eta_{5}^{2}-\eta_{1}^{2} \eta_{5}^{2}-\eta_{4}^{2} \eta_{6}^{2}\right|}{2 \eta_{1} \eta_{4} \eta_{5} \eta_{6}} \\
\left|\cos \left(\phi_{6}-\phi_{4}+\phi_{5}-\phi_{3}\right)\right| & =\frac{\left|\eta_{2}^{2} \eta_{6}^{2}-\eta_{4}^{2} \eta_{5}^{2}-\eta_{3}^{2} \eta_{6}^{2}\right|}{2 \eta_{3} \eta_{4} \eta_{5} \eta_{6}} .
\end{aligned}
$$

Equations (2)-(10) set boundaries for physically possible coupling amplitudes and phases of the generic lossless $3 p$ beam splitter-combiner. Equations (2)-(4) represent the energy conservation law and arise from the diagonal elements of the unitarity condition. Equations (5)-(10) arise from the off-diagonal elements. They are already simplified to contain just a single cosine term. However, it can be easily deduced that up to three phases in the scattering matrix $\mathbf{S}$ can be chosen arbitrarily. In this analysis we choose the phases $\phi_{1}, \phi_{2}, \phi_{3}$ to be zero. This is a permitted choice without introducing any restriction on possible coupling amplitudes. Then the phases of the scattering matrix can be written as

$$
\begin{aligned}
& \phi_{1}=\phi_{2}=\phi_{3}=0, \\
& \phi_{4}=-\frac{1}{2} \arccos \left(\frac{\eta_{1}^{2} \eta_{4}^{2}+\eta_{2}^{2} \eta_{4}^{2}-\eta_{5}^{2} \eta_{6}^{2}}{2 \eta_{4}^{2} \eta_{1} \eta_{2}}\right)-\frac{\pi}{2},
\end{aligned}
$$

$$
\begin{aligned}
& \phi_{5}=\frac{1}{2} \arccos \left(\frac{\eta_{4}^{2} \eta_{6}^{2}-\eta_{1}^{2} \eta_{5}^{2}-\eta_{3}^{2} \eta_{5}^{2}}{2 \eta_{5}^{2} \eta_{1} \eta_{3}}\right), \\
& \phi_{6}=-\frac{1}{2} \arccos \left(\frac{\eta_{2}^{2} \eta_{6}^{2}+\eta_{3}^{2} \eta_{6}^{2}-\eta_{4}^{2} \eta_{5}^{2}}{2 \eta_{6}^{2} \eta_{2} \eta_{3}}\right)+\frac{\pi}{2} .
\end{aligned}
$$

It is interesting to note that the coupling relations restrict the possible values of $\eta_{i}$. Let us assume that a free choice of $\eta_{4}^{2}$ and $\eta_{6}^{2}$ is desired, which then immediately determines $\eta_{2}^{2}$ according to Eq. (3). Substituting $\eta_{1}$ and $\eta_{3}$ by using Eqs. (2) and (4), Eqs. (5)-(10) provide the following pair of inequalities that restricts the values of $\eta_{5}$ and thereby also the values of $\eta_{1}$ and $\eta_{3}$ :

$$
\frac{\eta_{4} \eta_{6}\left(1-\eta_{2}\right)}{\eta_{4}^{2}+\eta_{6}^{2}} \leqslant \eta_{5} \leqslant \frac{\eta_{4} \eta_{6}\left(1+\eta_{2}\right)}{\eta_{4}^{2}+\eta_{6}^{2}} .
$$

We now apply a $3 p$ beam splitter-combiner in interferometry. We focus on the device in Fig. 1(b) as a coupler to a Fabry-Perot ring cavity as shown in Fig. 2. Laser light incident from the left is coupled according to $\eta_{4}^{2}$ into the cavity, which is formed by the grating and two additional highly reflecting cavity mirrors. If both cavity mirrors are lossless, the cavity finesse depends on the specular reflectivity $\eta_{2}^{2}$ and does not rely on high values of first- or second-order diffraction efficiencies. Using high reflection dielectric coatings makes high-finesse values and high laser buildups possible, similar to the linear cavity investigated in Ref. 1 . However, here the cavity outputs depend on $\eta_{4}^{2}$ (into port $c_{1}$ ) and $\eta_{6}^{2}$ (into port $c_{3}$ ) that can have different values.

Assuming unity laser input and perfectly reflecting cavity mirrors, the system is described by

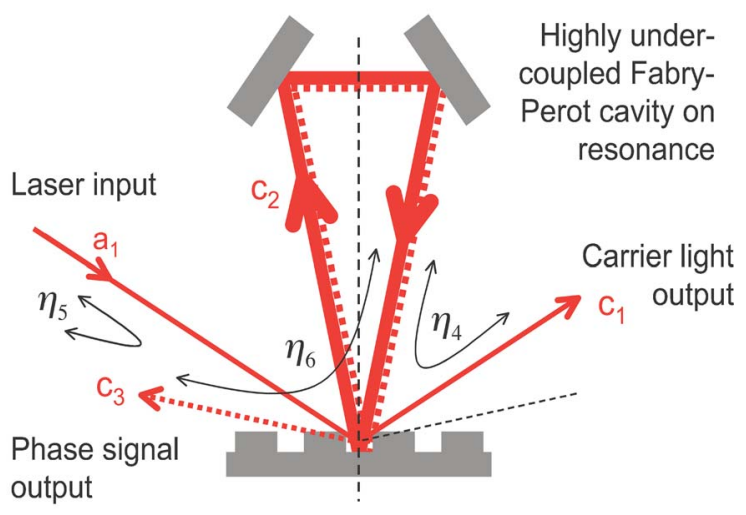

Fig. 2. (Color online) Three-port coupled grating in a ring Fabry-Perot interferometer. The grating can be designed such that the laser input is completely sent into port $c_{1}$ on cavity resonance. If the cavity is impedance matched this device might serve as an all-reflective mode cleaner. Another interesting case occurs in which the cavity is highly undercoupled. Then almost the complete cavity strain signals are sent to port $c_{3}$. Such a device separates carrier light from its modulation sidebands. 


$$
\left(\begin{array}{l}
c_{1} \\
c_{2} \\
c_{3}
\end{array}\right)=\mathbf{S} \times\left(\begin{array}{c}
1 \\
c_{2} \exp (2 i \theta) \\
0
\end{array}\right) .
$$

Here $\theta=\omega L / c$ denotes the detuning from cavity resonance, with $L$ the cavity length, $\omega$ the laser field angular frequency, and $c$ the speed of light. Solving for the reflected amplitudes yields

$$
\begin{aligned}
& c_{1}=\eta_{1}+\frac{\eta_{4}^{2} \exp \left[2 i\left(\phi_{4}+\theta\right)\right]}{1-\eta_{2} \exp (2 i \theta)}, \\
& c_{2}=\frac{\eta_{4} \exp \left(i \phi_{4}\right)}{1-\eta_{2} \exp (2 i \theta)}, \\
& c_{3}=\eta_{5} \exp \left(i \phi_{5}\right)+\frac{\eta_{4} \eta_{6} \exp \left[i\left(\phi_{4}+\phi_{6}+2 \theta\right)\right]}{1-\eta_{2} \exp (2 i \theta)} .
\end{aligned}
$$

From Eq. (14) it can be shown that, for a grating with $\eta_{5}^{2}$ at its maximum value for given $\eta_{4}^{2}$ and $\eta_{6}^{2}$, and a cavity on resonance $(\theta=0)$, no carrier light from the laser incidenting from the left is leaving the cavity to the left $\left(c_{3}=0\right)$. This dark port is indicated in Fig. 2 by a dashed arrow. If the cavity moves away from resonance, for example, caused by a cavity strain, amplitude $c_{3}$ is no longer zero. This field is generally termed a phase signal and might appear at some sideband frequency $\Omega$ if the cavity is locked to the time-averaged carrier frequency $\omega_{0}$ with a locking bandwidth smaller than $\Omega$. The phase signal generated inside the cavity obviously leaves the cavity according to the magnitudes of $\eta_{4}^{2}$ and $\eta_{6}^{2}$ in two directions. From Eqs. (14) and (16) it is easy to prove that the power of the signal indeed splits according to the ratio $\eta_{4}^{2} / \eta_{6}^{2}$. We now discuss two distinct examples; in both of them we consider $\eta_{5}^{2}$ to be designed close to its maximum value. For $\eta_{4}^{2}=\eta_{6}^{2}$ the cavity output coupling is twice the input coupling and the signal is split into two equal halves. We term this case a symmetric or an impedance-matched $3 p$ coupled cavity; this is in analogy to the lossless impedancematched linear cavity whose output coupling is also twice the input coupling. However, due to the choice of $\eta_{5}^{2}$ all the carrier power is sent into port $c_{1}$ if the cavity is on resonance as discussed above. Such a device can serve as an all-reflective mode cleaner. For $\eta_{4}>\eta_{6}$ the $3 \mathrm{p}$ coupled lossless cavity can be termed overcoupled and for $\eta_{4}<\eta_{6}$ undercoupled. As the second example we consider the highly undercoupled grating cavity $\left(\eta_{4}^{2} \ll \eta_{6}^{2} \ll \eta_{2}^{2}\right)$ and explicitly choose the following coupling coefficients:

$$
\begin{aligned}
& \eta_{4}^{2}=0.0001, \quad \eta_{6}^{2}=0.0099, \quad \eta_{2}^{2}=0.99, \\
& \eta_{5}^{2}=0.0394, \quad \eta_{1}^{2}=0.9605, \quad \eta_{3}^{2}=0.9507, \\
& \phi_{1}=0, \quad \phi_{2}=0, \quad \phi_{3}=0 \text {, } \\
& \phi_{4} \approx-3.1349, \quad \phi_{5} \approx 1.5708, \quad \phi_{6} \approx 1.5707 \text {. }
\end{aligned}
$$

For this set of measures again $\eta_{5}^{2}$ is almost at its maximum value, and consequently $\eta_{1}^{2}$ and $\eta_{3}^{2}$ are close to their minimum values. As in the impedancematched case described above, again all the carrier power is sent into port $c_{1}$. Owing to the high asymmetry of the ratio between $\eta_{4}^{2}$ and $\eta_{6}^{2}$ the signal is sent mainly into port $c_{3}$. The special property of the highly undercoupled grating Fabry-Perot interferometer is therefore the possibility of separating carrier light and phase signal. This is a remarkable result. Separation of carrier light and phase signal is well known for a Michelson interferometer operating on a dark fringe. Such an interferometer sends all the laser power back to the laser source. The antisymmetric mode of phase shifts in the Michelson arms is sent into the dark port. The symmetric mode is combined with the reflected laser power and sent toward the bright port. In the case of the highly undercoupled $3 \mathrm{p}$ grating Fabry-Perot interferometer the almost complete phase signal is separated from carrier light and is accessible to detection, and the reflected field in the bright port contains only a marginal fraction of the signal $\left(\eta_{4}^{2} / \eta_{6}^{2}\right)$.

We point out that all results obtained for the Fabry-Perot ring interferometer using the $3 p$ coupler in Fig. 1(b) also hold for a linear cavity using the $3 p$ coupler in Fig. 1(a). However, some distinctive properties should be mentioned. Regardless of their different topologies, the ring Fabry-Perot interferometer is content with only low efficiencies for greater than zero diffraction orders. All coupling amplitudes in Eqs. (17) with values close to unity describe specular reflections. The production of such a grating with low overall loss should be possible with standard technologies building on the concept used in Refs. 1 and 4 . In the case of the (highly undercoupled) linear Fabry-Perot interferometer $\eta_{1}^{2}$ and $\eta_{3}^{2}$ do not describe specular reflections, and high diffraction efficiencies in the second-order diffraction are required. However, especially in the second-order Littrow configuration, carrier and signal separation offers straightforward extension by interferometer recycling techniques. ${ }^{5}$

This work was supported by the Deutsche Forschungsgemeinschaft within the Sonderforschungsbereich TR7. R. Schnabel's e-mail address is roman.schnabel@aei.mpg.de.

\section{References}

1. A. Bunkowski, O. Burmeister, P. Beyersdorf, K. Danzmann, R. Schnabel, T. Clausnitzer, E.-B. Kley, and A. Tünnermann, Opt. Lett. 29, 2342 (2004).

2. A. Bunkowski, O. Burmeister, K. Danzmann, and R. Schnabel, Opt. Lett. 30, 1183 (2005).

3. A. E. Siegmann, Lasers (University Science Books, 1986), p. 401.

4. T. Clausnitzer, E.-B. Kley, A. Tünnermann, A. Bunkowski, O. Burmeister, K. Danzmann, R. Schnabel, A. Duparré, and S. Gliech, Opt. Express 13, 4370 (2005).

5. G. Heinzel, K. A. Strain, J. Mizuno, K. D. Skeldon, B. Willke, W. Winkler, R. Schilling, A. Rüdiger, and K. Danzmann, Phys. Rev. Lett. 81, 5493 (1998). 